\title{
Evaluating the Impact of Mediated Literature Searches at a Comprehensive Cancer Center
}

\author{
Donna S. Gibson, Antonio P. DeRosa, Marisol Hernandez \& Konstantina \\ Matsoukas
}

To cite this article: Donna S. Gibson, Antonio P. DeRosa, Marisol Hernandez \& Konstantina Matsoukas (2017) Evaluating the Impact of Mediated Literature Searches at a Comprehensive Cancer Center, Medical Reference Services Quarterly, 36:3, 229-239, DOI: 10.1080/02763869.2017.1332146

To link to this article: https://doi.org/10.1080/02763869.2017.1332146

\section{册 Published online: 17 Jul 2017.}

\section{Submit your article to this journal $\sqrt{3}$}

Џ Article views: 180

View Crossmark data ¿ 


\title{
Evaluating the Impact of Mediated Literature Searches at a Comprehensive Cancer Center
}

\author{
Donna S. Gibson, Antonio P. DeRosa, Marisol Hernandez, and \\ Konstantina Matsoukas
}

Nathan Cummings Center, Medical Library, Memorial Sloan Kettering Cancer Center, New York, New York, USA

\begin{abstract}
Research informationists at a comprehensive cancer center sought to evaluate the impact and value of mediated literature searches in support of their users' work activities. An assessment tool was identified in the literature and modified by the investigators to solicit feedback from library users and identify the major reason(s) why scientists and health care professionals request literature searches, how they use the resulting information, and the impact that the results may or may not have on their research or patient care/decision-making activities. Survey results were qualitatively analyzed, and future avenues of outreach and promotion of mediated literature search services were identified.
\end{abstract}

\section{ARTICLE HISTORY}

Received 26 February 2017 Revised 30 March 2017 Accepted 18 April 2017

\section{KEYWORDS}

Assessment tool; comprehensive cancer center; literature search services; mediated literature searches; reference; research informationist; service impact; survey

\section{Introduction}

Gathering metrics and creating assessment tools that help demonstrate a library's value and impact are not unique to medical/research libraries. Many libraries collect various usage statistics and seek appropriate opportunities to demonstrate the need and importance of the services offered to their user community. It has been 25 years, however, since the landmark research study by Joanne G. Marshall was published ${ }^{1}$ and ten years since the National Network of Libraries of Medicine/Middle Atlantic Region (MAR) began efforts to replicate this type of study ${ }^{2}$ that evaluated the impact and value of library/information services on patient care and clinical decision-making. Over the last decade, the many uncertainties of the health care and economic environments have made organized "value" initiatives by library professional associations ${ }^{3}$ and the formal investigation of the impact of library and information services more of a priority, as demonstrated by the growing body of literature in this area. ${ }^{4-6}$ Although they provide useful evidence, the findings of these research studies cannot be entirely generalized to all environments.

CONTACT Donna S. Gibson gibsond@mskcc.org N Nathan Cummings Center, Medical Library, Memorial Sloan Kettering Cancer Center, 1275 York Avenue, New York, NY 10065, USA.

This article is based on a poster presented at the Annual Meeting of the Medical Library Association, Toronto, Canada, May 16, 2016. 
Therefore, in 2015 the Reference and Consultation Services team at Memorial Sloan Kettering (MSK) Library, a specialized cancer center library, decided to conduct a local "value" study in order to explore the impact, even beyond just patient care, of one key MSK library service-mediated literature searching.

The MSK Library supports one of the oldest cancer centers in the country. The institution was one of the first to receive the National Cancer Institute's Comprehensive Cancer Center designation with state-of-the-art research being conducted in tandem with quality patient care. ${ }^{7}$ The MSK Library's mission is to proactively partner with library users by delivering innovative services and targeted published content in support of quality patient care, research excellence, and ongoing learning for the progressive control and cure of cancer. An online assessment tool that had been designed to evaluate the impact of literature searches only on patient care $^{8}$ was adapted in order to broaden the scope of the assessment to include the evaluation on research, teaching/learning, and beyond.

Reference and consultation is a client-facing service and the primary point of contact for users in most libraries, whether in person or online. One of the key service functions for the reference librarian or research informationist at MSK is conducting mediated literature searches, primarily received as an online request with a prespecified due date via a web-based form. Researching a specific topic, locating relevant published literature, analyzing the results, and delivering the information electronically in a structured format are all components of a literature search service that generally save the time of health care professionals and researchers, allowing them to instead prioritize their other work activities. This article will share the findings of this local study that focused specifically on evaluating the impact of mediated literature searches (without the provision of any database search skill training/instruction), rather than investigate the impact of overall library resources and services support, as other recent studies have done. ${ }^{6}$

\section{Methods}

The MSK Library's Reference and Consultation Services team developed a nine-question survey by adapting a published assessment tool and using SurveyMonkey ${ }^{9}$ to design the online version of the survey instrument. The survey used was modified from a quick-assessment tool $^{8}$ that was previously used to evaluate the impact of literature searching services on patient care in an integrated health system located in and around Regina, Saskatchewan, Canada. ${ }^{8}$ The MSK Library wanted to investigate all potential reasons for a literature request, not just patient care, and how the information was leveraged by the user, so the tool was adapted accordingly. The modified survey's questions explored the reason(s) for the search, the user's plans for using the information received, and the potential impact on the user's work. Its 
intended audience included physicians, nurses, researchers, interns, research fellows, students, and other health care professionals.

Each time a literature search request was received, when it came time to send the results, the reference team (three librarians) sent the survey link to the nine-question survey via email along with the search results, as well as a brief explanation on the study's data collection goals and what the team hoped to accomplish with the solicited feedback. Data collection started in February 2015 and ended in December 2015. Survey respondents were asked to base their replies on the search results received and not reflect on a past interaction with one of the reference librarians or a past literature search request. This approach was done to capture respondents' immediate reactions to the information delivered and avoid any memory bias or possible difference in recall of the impact and purpose of the search results. Repeat users were permitted and encouraged to submit a survey response for each literature search requested.

\section{Survey Findings}

During the 11-month period of data gathering, 805 searches were delivered. From this pool of potential survey responses, the team received a total of 220 completed surveys, for a survey response rate of $27.3 \%$.

The survey (see the Appendix for survey questions) began by asking the respondents where they fit within the institution, followed by the purpose for which they were requesting a search. The majority of individuals who completed the survey were internal users, with only one patient/caregiver taking the time to provide their assessment of the search results delivered. Patients, their families, and caregivers represent fewer than $5 \%$ of the overall search requests received each month. Most requests from this audience focus on asking for a specific published paper or scholarly article.

The second question offered a selection of possible answers for the purpose of the search request, and respondents were asked to select the most appropriate answer. The top four choices included research, meeting presentations, publication/report, and self-continuing education. Patient care was the fifth most frequently selected response out of the eight options listed. When evaluating the responses for this question, there were 30 responses under "Other," which were later redistributed to their most appropriate category by the research team. To better represent the overall output, these responses were sorted based on the answers provided. This exercise led to one answer choice being expanded to include reports (publication/report) and to two new answer choices being created to accommodate the responses (project, resume/CV). In the end, there were nine answer choices developed following the analysis of the survey data received (see Table 1). This better represented the data breakdown for this question. The research team did not need to re-code any of the other questions and responses. 
Table 1. Search request purpose*.

\begin{tabular}{lr}
\hline Answer Choices & Response Count (\%) \\
\hline Research & $80(35.4)$ \\
Meeting presentation & $45(20.0)$ \\
Publication/Report & $29(12.8)$ \\
Continuing education (self) & $23(10.2)$ \\
Patient care & $21(9.2)$ \\
Project & $13(5.7)$ \\
Teaching & $7(3.1)$ \\
Grant writing & $5(2.2)$ \\
Resumé (CV) & $3(1.3)$ \\
\hline
\end{tabular}

*Re-coded 30 responses where "other" was selected as an answer choice.

The next survey question teased out whether it was the respondent's first time requesting a literature search. Approximately two-thirds (66.36\%) of respondents indicated this was not their first time submitting a request. Five respondents $(2.27 \%)$ could not remember if they had submitted a previous request, and $69(31.36 \%)$ indicated it was their first literature search request submission.

Question 4 was multiple-choice: Did the information received from the MSK Library answer your question? Answer options were "Yes," "No," or "Partially." Two hundred and four respondents (92.73\%) selected "Yes," one indicated "No" (0.45\%), and 15 selected "Partially" (6.82\%).

The next question listed potential answer choices for the immediate impact of the information that the user received on their work or research activity. Respondents could select all answers that applied to the search request. The majority of respondents agreed that the information sent had some level of impact (14 indicated "no immediate impact," and two selected from the list that the information was "not received in time to have an immediate impact"). Table 2 illustrates, from most frequent to least, respondents' selections from the answer choices available to them. The top five answers were: 1) Helped me to generate new ideas and insights; 2) Helped me with my presentation or talk; 3) Supported my current research/rethink my research; 4) Saves me time; and 5) Keeps me updated in my area of expertise.

The answer options offered were grouped during the analysis into two larger categories to distinguish what could be considered user-related impact or patient-related impact. The top five answers selected all fell under userrelated impact. It is extremely difficult to place a value on patient-related impact. While the frequency of the answer options were smaller, response selection revealed that the research informationist did support patient care by providing information that either changed a respondent's productivity or decision-making, or altered how the individual approached patient care. Patient-related impact answers included:

- Helps to increase my productivity (27\%);

- Information was used to enhance/improve a MSK procedure/process/ policy $(23 \%)$; 
Table 2. Immediate impact of information on work or research activity.

\begin{tabular}{lr}
\hline & Response \\
Answer Choices (Respondents could select multiple answers) & Count (\%) \\
\hline Helped me to generate new ideas and insights & $109(49.55)$ \\
Helped me with my presentation or talk & $109(49.55)$ \\
Supported my current research/rethink my research & $108(49.09)$ \\
Saves me time & $96(43.64)$ \\
Keeps me updated in my area of expertise & $94(42.73)$ \\
Helps to increase my productivity & $60(27.27)$ \\
Information was used to enhance/improve a MSK procedure/process/policy & $50(22.73)$ \\
Helps me to make better decisions & $49(22.27)$ \\
Confirmed the knowledge/expertise I have & $49(21.82)$ \\
Treatment/management (confirmed/changed drugs, treatment, and/or post care plan) & $19(8.64)$ \\
Refreshed memory & $17(7.73)$ \\
There was no immediate impact & $14(6.36)$ \\
Saves my department money & $12(5.45)$ \\
Diagnosis (confirmed/changed diagnosis and/or diagnostic test/procedure) & $7(3.18)$ \\
Avoided adverse event(s)/critical incident(s) & $6(2.73)$ \\
Prevented referral to or consultation by another department & $5(2.27)$ \\
Initiated referral to or consultation by another department & $5(2.27)$ \\
Information was not received in time to have an immediate impact & $2(0.91)$ \\
\hline
\end{tabular}

- Helps me to make better decisions (22\%);

- Treatment/management (confirmed/changed drugs, treatment, and/or post care plan) (9\%);

- Diagnosis (confirmed/changed diagnosis and/or diagnostic test or procedure) (3\%);

- Avoided adverse event(s)/critical incident(s) (3\%);

- Initiated referral to or consultation by another department (2\%); and

- Prevented referral to or consultation by another department (2\%).

There was an option for respondents to provide an answer not listed. For this question, 17 comments were given, most complementing the work done by the research informationist and not relevant to immediate impact of information.

Question 6 asked the survey recipient to indicate why the information provided by the library did not answer their research question. Multiple answers could be selected. Out of the 220 who completed this survey, 57 skipped this question. With the remaining 163 respondents, a total of 169 answers were provided. The majority of replies fell under the answer choice "My question was answered" (87.12\%). The next most frequent answer was "There appears to be not enough evidence published on the topic (8.59\%). There was also an opportunity for respondents to leave a comment. Nine comments were shared, the majority thanking the librarians for the research provided or indicating the information was useful.

The following question centered on how the respondent was planning on using the information in the future (see Table 3). This was also a question where multiple answers could be selected. Only ten responses indicated the 
Table 3. Mediated search results: How will information be used?

\begin{tabular}{lr}
\hline Answer Choices (Respondents could select multiple answers) & Response Count (\%) \\
\hline Share/discuss with colleagues & $133(60.45)$ \\
Research or publish & $122(55.45)$ \\
Teach & $72(32.73)$ \\
Revision of clinical pathways, practice guidelines, policies, or procedures & $47(21.36)$ \\
Change approach to a particular patient and/or future patient(s) & $34(15.45)$ \\
Apply for grant funding & $29(13.18)$ \\
The information will not be used in the future & $10(4.55)$ \\
\hline
\end{tabular}

information would not be used in the future. The top three preferred answers were 1) share/discuss with colleagues, 2) research or publish, and 3) teach. There was an option for respondents to provide an answer not listed. For this question, 14 comments were given, most praising and showing appreciation for the results sent by the research informationist and not relevant to how the information will be used in the future.

Question 8 solicited their role at MSK, and respondents were asked to pick a title that best described their primary job responsibilities. The top three roles were nurse (96), clinician/physician (30), and research scientist (19). Tied for fourth place was administrative staff (manager, supervisor) and administrative support (assistant or secretary) (15 each).

The final question was asked for those respondents who might be willing to provide their contact information should additional information need to be gathered. Follow-ups were not conducted during or at the end of the survey; however, these individuals would be included in any future announcements regarding reference and consultation services.

\section{Discussion}

Consistent with the findings of other studies, ${ }^{2,8}$ the MSK survey results did indicate that the literature searches had an impact on patient care, with many of the survey respondents feeling that the information received either enhanced or improved an MSK procedure, touched patient treatment options, or helped with better clinical decision-making. The majority of respondents (92.73\%) agreed that the information received had some level of impact, and after an analysis of the survey results, other points of interest were revealed. Approximately $95 \%$ of respondents used the information they received to enhance their own knowledge base or to make a decision regarding patient care, suggesting that the reference librarians' intermediary searching expertise did indirectly contribute to patient care activities.

Most noteworthy, the survey results also suggested that providing a mediated literature search service, in support of the institution's mission in the areas of research excellence and ongoing learning, also has an impact on these activities. The search results received reportedly helped respondents 
to generate new ideas and insights, supported current research, kept users up-to-date in their area of expertise, and supported many of their scholarly activities. Grouped by theme, $77 \%$ of selected responses showed that the search results impacted or fostered scholarly communications in some way.

More than $66 \%$ of respondents shared that this was not their first time submitting a search request. Repeat business can speak to the level of trust users have with the quality of service offered and delivered. Repeat business helps to develop, enhance, and strengthen client relationships and provides opportunities for the research informationists to learn more about what matters to their users, placing them in a position to be more proactive and targeted in their client interactions.

\section{Conclusion}

The survey results confirmed for the library that at least in the specialized, local MSK environment, community members at various levels, not just physician researchers, are increasingly engaging in research and quality improvement projects in addition to patient care. Therefore, helping them find the research evidence they need via mediated literature searches remains a key library service worthy of further investigation. Looking to the future, there is an opportunity to reach out to those survey respondents who provided their contact information to more deeply explore the benefits of mediated literature searches. Face-to-face interviews could provide another dimension of responses that may perhaps complement the survey results and provide additional insights on the added value of mediated literature searches, as well as identify ways to further enhance this service for researchers and health care professionals. This research would not only help inform the decision-making process of library managers but could also lead to more robust data-driven promotional and marketing messages. Ultimately, the benefits of leveraging the librarians' searching and research expertise could be used to attract new customers to use this library service and to improve the library's impact and value for patient care and clinical decision-making.

\section{Acknowledgments}

A special thank you to Ashley Farrell (information specialist, University Health Network, Toronto, Ontario, Canada) and Jeff Mason (horizon scanning officer, Canadian Agency for Drugs and Technologies in Health, Ottawa, Ontario, Canada), who encouraged other librarians to further validate and use their survey tool.

\section{Notes on Contributors}

Donna S. Gibson, MLS (gibsond@mskcc.org) is Director of Library Services, Nathan Cummings Center, Medical Library, Memorial Sloan Kettering Cancer Center, 1275 York Avenue, New York, NY 10065. 
Antonio P. DeRosa, MDS, MLIS, AHIP (derosaa1@mskcc.org) is Research Informationist, Nathan Cummings Center, Medical Library, Memorial Sloan Kettering Cancer Center, 1275 York Avenue, New York, NY 10065.

Marisol Hernandez, MLS, MA (hernanm4@mskcc.org) is Research Informationist, Nathan Cummings Center, Medical Library, Memorial Sloan Kettering Cancer Center, 1275 York Avenue, New York, NY 10065.

Konstantina Matsoukas, MLIS (matsoukk@mskcc.org) is Research Informationist, Nathan Cummings Center, Medical Library, Memorial Sloan Kettering Cancer Center, 1275 York Avenue, New York, NY 10065.

\section{References}

1. Marshall, Joanne G. "The Impact of the Hospital Library on Clinical Decision Making: The Rochester Study." Bulletin of the Medical Library Association 80, no. 2 (April 1992): 169-178.

2. Marshall, Joanne G., Julia Sollenberger, Sharon Easterby-Gannett, et al. "The Value of Library and Information Services in Patient Care: Results of a Multisite Study." Journal of the Medical Library Association 101, no. 1 (January 2013): 38-46.

3. Bandy, Margaret Moylan. "Pivoting: Leveraging Opportunities in a Turbulent Health Care Environment." Journal of the Medical Library Association 103, no. 1 (January 2015): 3-13.

4. Marshall, Joanne G., Jennifer C. Morgan, Cheryl A. Thompson, and Amber L. Wells. "Library and Information Services: Impact on Patient Care Quality." International Journal of Health Care Quality Assurance 27, no. 8 (2014): 672-683.

5. Perrier, Laure, Ann Farrell, A. Patricia Ayala, et al. "Effects of Librarian-provided Services in Healthcare Settings: A Systematic Review." Journal of the American Medical Informatics Association 21, no. 6 (November 2014): 1118-1124.

6. Sollenberger, Julia F., and Robert G. Holloway, Jr. "The Evolving Role and Value of Libraries and Librarians in Health Care." JAMA 310, no. 12 (September 2013): 1231-1232.

7. National Cancer Institute. "NCI-Designated Cancer Centers." Accessed January 13, 2017. https://www.cancer.gov/research/nci-role/cancer-centers/find/memorialsloankettering/.

8. Farrell, Ashley, and Jeff Mason. "Evaluating the Impact of Literature Searching Services on Patient Care Through the Use of a Quick-Assessment Tool." Journal of the Canadian Health Libraries Association / Journal de l'Association des bibliothèques de la santé $d u$ Canada 35, no. 3 (2014): 116-123.

9. SurveyMonkey. Accessed January 13, 2017. https://www.surveymonkey.com/.

\section{Appendix: Survey Questions}

\section{Value of Mediated Literature Searching}

Results from a literature search conducted on your behalf were recently sent to you. The reference team would like to evaluate the impact these searches have on your work in order to look for possible opportunities to improve this service. Please take a moment to share your thoughts about the outcome of your search request. The survey has been designed to obtain feedback quickly and minimize the time required to complete it. 
If you have completed this survey before (following a previous search request), please take a moment to complete it again as the questions pertain to an individual literature search (not the search service as a whole).

* 1 . Select the area where you best fit.....

Memorial Sloan Kettering Cancer Center

Memorial Hospital

Sloan Kettering Institute

Patient/Caregiver

Visitor

*2. What was the purpose of requesting your literature search? (select the most appropriate answer)

Continuing education (self)

Research

Patient care

Grant writing

Teaching

Meeting presentation

Publication

Other (please specify)

* 3. Is this your First time submitting a literature search request?

Yes

No

Don't remember/recall

* 4. Did the information you received from the Memorial Sloan Kettering Library answer your question?

Yes

No

Partially

* 5. What was the immediate impact of the information you received from the Memorial Sloan Kettering Library on your work or research activity? Please select all that apply.

There was no immediate impact

Information was not received in time to have an immediate impact

Information was used to enhance/improve a MSK procedure/process/policy Diagnosis (confirmed/changed diagnosis and/or diagnostic test or procedure) Avoided ordering of additional/unnecessary diagnostic test(s)/procedure(s) Treatment/management (confirmed/changed drugs, treatment, and/or post care plan)

Avoided adverse event(s)/critical incident(s)

Prevented referral to or consultation by another department

Initiated referral to or consultation by another department

Helped me to generate new ideas and insights

Supported my current research/rethink my research 
Keeps me updated in my area of expertise

Refreshed memory

Confirmed the knowledge/expertise I have

Helped me with my presentation or talk

Helps to increase my productivity

Helps me to make better decisions

Saves me time

Saves my department money

Other (please specify)

6. Please indicate why the information provided by the Memorial Sloan Kettering Library *DID NOT* answer your question. Please select all that apply.

My question was answered

No answer exists

Information provided did not answer the question

Information was not received on time

Information was received on time but not enough time to read it

Information provided was not on target

Results sent were not "evidence-based"

There appears to be not enough evidence published on the topic

Other (please specify)

* 7. Regardless of immediate impact or completeness of answer, how will you use the information provided by the Memorial Sloan Kettering Library in the future? Please select all that apply.

The information will not be used in the future

Change approach to a particular patient and/or future patient(s)

Share/discuss with colleagues

Teach

Research or publish

Apply for grant funding

Revision of clinical pathways, practice guidelines, policies or procedures

Other (please specify)

* 8. Please tell us a bit about yourself. What best describes your primary job duties?

Administrative support (assistant, secretary)

Administrative staff (manager, supervisor)

Clinician/Physician

Fellow

Health care professional

Intern

Lab Head

Nurse 
Patient/Caregiver

Pharmacist

Research assistant

Research fellow

Research scientist

Resident

Student

Visiting Investigator

Visitor

9. Thank you for providing your feedback. It is only with your help that we can improve the service we offer. Please provide your contact information as we may wish to follow up with you. If you are selected, you will be invited to participate in a brief conversation later in the year. Name:

Email:

Phone: 